\title{
Treatment of onychomycosis using a 1064-nm diode laser with or without topical antifungal therapy: a single-center, retrospective analysis in 56 patients
}

\author{
G. C. Weber ${ }^{1}$, P. Firouzi ${ }^{1}$, A. M. Baran ${ }^{1}$, E. Bölke ${ }^{2}$, H. Schrumpf ${ }^{1}$, B. A. Buhren ${ }^{1}$, B. Homey ${ }^{1}$ and P. A. Gerber ${ }^{{ }^{*}}$
}

\begin{abstract}
Background: Currently available treatment options for onychomycosis such as topical and systemic antifungals are often of limited efficacy, difficult to administer or associated with relevant side effects. Non-ablative laser therapy is proposed to represent a safe alternative without the disadvantages of drugs. Yet, to date, the efficacy of laser therapy for onychomycosis is discussed controversially. Against this background, we performed a systematic retrospective analysis of our clinical experience of 4 years of onychomycosis treatment applying a long-pulsed 1.064-nm diode laser.
\end{abstract}

Methods: We retrospectively evaluated the records of 56 patients with microscopic and culturally proven onychomycosis affecting a toenail of the hallux and other toes, who had been treated with a long-pulsed 1.064-nm diode laser (FOX, A.C.R. Laser GmbH, Nuremberg) during the time period of July 2013-December 2016 with or without concomitant topical antifungals. Thereof, 27 patients received laser treatment and 29 patients received laser treatment in combination with local antifungals. We conducted a mean of 3.9 laser treatments at 2-6-week intervals. The primary endpoint of our analysis was clinical improvement; secondary endpoints were complete remission of fungal pathogens in fungal culture and in microscopy.

Results: Clinical improvement was achieved in $56 \%$ of patients treated with laser only after a mean of 4.5 treatments and in $69 \%$ of patients treated with laser in combination with topical antifungals after a mean of 3.6 treatments. Cultural healing was detected in $63 \%$ of patients treated with laser only after a mean of 5.4 treatments, vs. $86 \%$ of patients treated with laser and concomitant topical antifungals after a mean of 4.8 treatments. Microscopic healing (complete healing) with the absence of fungal pathogens was achieved in $11 \%$ of patients after a mean of 4.7 treatments with laser only, vs. $21 \%$ of patients treated with laser and concomitant topical antifungals after a mean of 4 treatments. No relevant adverse effects were observed.

Conclusions: The 1.064-nm diode laser is an effective and safe option for the treatment of onychomycosis. Of note, the combination with topical antifungals will increase overall treatment efficacy and reduce the time to healing. Particularly, patients with contraindications against systemic antifungals may benefit from this multimodal therapeutic approach. Our data, moreover, suggest that treatment efficacy is positively correlated with the total number of laser treatments.

\footnotetext{
*Correspondence: peterarne.gerber@med.uni-duesseldorf.de

1 Department of Dermatology, Heinrich-Heine-University,

40225 Düsseldorf, Germany

Full list of author information is available at the end of the article
} 


\section{Background}

Onychomycosis is one of the most prevalent nail disorders in adults affecting $4-8 \%$ of the general population worldwide $[1,2]$. It is a fungal nail infection primary caused by dermatophytes [3]. The presence of previous nail trauma, diabetes mellitus and advancing age, as well as restricted peripheral circulation represent risk factors for onychomycosis [4-6]. Finally, in particular, patients with compromised immune function are at an increased risk of fungal nail diseases and are susceptible to secondary infections such as cellulitis or generalized tinea corporis. The main clinical characteristics of onychomycosis are focal parakeratosis, subungual hyperkeratosis with onycholysis (detachment of the nail plate from the nail bed) and thickening of the subungual region. Secondary superinfecting bacteria and nondermatophytic molds can give the nail plate a yellowish brown appearance. Advanced infections can then lead to total destruction of the nail plate $[7,8]$.

Current recommendations for the treatment of onychomycosis include the atraumatic extraction of onychomycotic nail material, e.g. via application of topical urea, and local or systemic antifungals [9]. Yet, available treatments are limited by moderate efficacy or other restraints. Topical antifungals such as amorolfine, ciclopiroxolamine or terbinafine nail lacquer often take a long time to eradicate the infection and barely penetrate the nail plate at fungicidal concentrations $[10,11]$. Systemic antifungals such as fluconazole, itraconazole or terbinafine have been associated with relevant side effects such as congestive heart failure, hepatotoxicity and systemic drug interactions $[4,12,13]$. To conclude, long duration of treatment, bad compliance, severity of side effects or simply patient refusal of a systemic therapy, as well as patients not responding to treatment represent the main challenges in onychomycosis therapy.

Against this background, almost a decade ago nonablative laser therapy was introduced as a novel treatment option for onychomycosis. In 2010, the FDA approved the use of a 1.064-nm Nd:YAG laser for this indication [14]. Since then, a broad range of different laser systems for the treatment of onychomycosis were introduced into the market [15].

Whereas the definite mode of action of non-ablative lasers for onychomycosis therapy remains unclear, one current hypothesis is the selective photothermolysis of pigments of dermatophytes with a consecutive generation of temperatures of greater than $50{ }^{\circ} \mathrm{C}$. This heating is proposed to cause the destruction of fungal structures and thereby to eradicate the fungal infection of the nail [16-22]. An alternate hypothesis proposes an unspecific effect of tissue heating with a subsequent increase in circulation due to vasodilatation and stimulation of an immunological cure of the infection [23].

Today, laser therapy of onychomycosis is discussed controversially. Several case series, comments and studies published in the past decade report divergent results and conclusions with regard to efficacy, adverse effects and safety or even necessity of the treatment [15, 24, 25]. Of note, a systematic assessment of these published data also shows great inhomogeneity with regard to treatment protocols, endpoints, techniques, time points of analysis, and many other factors, which makes it difficult to compare results and to support drawn conclusions. Taken together, it can, however, be concluded from published data that sole laser treatment is likely less effective than pharmacological therapies. To date, analyses assessing potential synergies of laser therapy in combination with topical or systemic antifungals are sparse [25]. It is obvious that, in particular, patients with contraindications against systemic antifungals could benefit from such a multimodal approach.

Here, we retrospectively analyzed clinical improvement and healing by assessing the records of 56 patients with onychomycosis of the toenails that were treated in our clinic with laser therapy alone or with laser therapy in combination with topical antifungals.

\section{Patients and methods}

In our retrospective analysis, we evaluated the records of all patients with proven onychomycosis caused by dermatophytes and affecting the toenails, including at least one hallux, which we treated with laser therapy in the time period of July 2013-December 2016. All patients enrolled in this study had a histologically and microbiologically proven diagnosis of onychomycosis caused by dermatophytes. We identified a total of 56 patients that fulfilled these criteria and included them in our analysis. In total, 27 patients received laser treatment alone and 29 patients received laser treatment plus topical antifungals (ciclopirox, or amorolfine nail lacquer or cream as indicated by the manufacturers).

The mean patient age was 62.4 years; 37 males (mean age $=64$ years; age range $=27-92$ years) and 19 females (mean age $=59$ years, age range $=25-76$ years) were included. Out of these 56 patients, 29 had already used topical antifungals (amorolfine or ciclopiroxolamine) for over 24 weeks and had reported that they did not observe any significant improvement prior to laser therapy. To standardize our analysis, we focused only on the toenail of one affected hallux. In all cases, fungal culture was positive for dermatophytes. In total, patients received an average rate of 3.9 laser sessions (range $=1-11$ ). During every visit clinical courses were documented by standardized photography. 
Treatment sessions were conducted every 2 weeks for the first 3 sessions and every 6 weeks thereafter. Laser treatment was performed with a 1.064-nm diode laser (FOX, A.R.C. Laser GmbH, Nuremberg) in pulsed mode with a spot size of $4 \mathrm{~mm}$. Additional laser settings were power $=8 \mathrm{~W}$, pulse duration $=80 \mathrm{~ms}$, and repetition rate $=5.6 \mathrm{~Hz}$. A total energy of $500-800 \mathrm{~J}$ was applied per session and great toenail depending on the treatment-associated pain. Prior to each laser session visible dystrophic nail material was mechanically removed by a podiatrist. During treatment, the laser beam was applied continuously with an average swipe speed of around $2-4 \mathrm{~mm} / \mathrm{s}$ over the entire area of the nail and nail bed in a grid pattern until the patient reported moderate to strong heat.

\section{Assessment of therapeutic endpoints}

Clinical and microbiological endpoints were systematically assessed. Patients' and physicians' evaluation of the therapeutic response was collected from the patients' records. In addition, an independent dermatologist evaluated all photographs that had been taken before every session and at the final visit.

The primary endpoint, clinical improvement, was defined as a significant clinical improvement evaluated by the patient himself and assessment of the photographic documentation by an independent physician. Clinical cure was defined as $100 \%$ clear nail with no subjectable clinical signs of fungal nail infection via assessment of the photographic documentation by the independent physician. Furthermore, at the end of the last laser treatment, a final fungal culture and microscopic assessment of mechanically removed nail material were performed. Secondary endpoints were accordingly defined as the clearance of fungal pathogens in (i) microscopic examination and (ii) in fungal culture after up to 4 weeks of incubation. Direct fluorescence microscopy assay was used for microscopic examination. Therefore, Blankophor was used as a fast fluorescent whitener and direct detection of fungal elements was determined using a Zeiss Axioplan fluorescence microscope.
Fungal growth and spore production in culture medium were performed on Sabouraud agar for up to 3 weeks at room temperature. Mycological cure was defined as both negative microscopy and negative culture.

\section{Results}

A total of 56 patients were treated with a 1.064-nm diode laser (FOX, A.R.C. Laser GmbH, Nuremberg) in pulse mode with a total energy of 500-800 J per nail of the hallux, depending on pain intensity.

\section{Number of treatments}

The average number of treatments was 3.9 (range 1-11). Eleven patients received 1 laser session, 22 patients received 2-4 sessions, and 23 patients received more than 5 sessions.

\section{Clinical results}

Table 1 shows the clinical results of the patients separated into groups. Figure 1 shows representative clinical courses. Patients treated with laser only received an average of 4.5 treatments. Patients treated with combination therapy received an average of 3.6 treatments. Significant subjective clinical improvement was achieved in 56\% $(n=15)$ treated only with laser after 5.1 laser treatments, and in $69 \%(n=20)$ treated in average 4.5 times with laser plus topical antifungals. For nails treated with laser only, complete clinical clearance was achieved in $26 \%$ as compared to $21 \%$ for nails treated with laser plus topical antifungals. For nails treated with laser plus topical antifungals, this result was achieved after an average 4.3 laser sessions as compared to 6.0 sessions for laser only. During therapy, besides mild pain and reported heat, no significant side effects were observed.

\section{Mycological results}

Table 2 shows the mycological results of the fungal culture. Table 3 shows the microscopic outcomes. The secondary endpoint "cultural cure" with no evidence of fungal growth in culture could be achieved in $75 \%$

Table 1 Clinical efficacy of laser plus/minus topical antifungals for the treatment of onychomycosis

\begin{tabular}{lllll}
\hline & $\begin{array}{l}\text { Average of laser sessions } \\
\text { (all patients) }\end{array}$ & Clinical improvement & $\begin{array}{l}\text { Average of laser } \\
\text { sessions }\end{array}$ & $\begin{array}{l}\text { Clinical cure } \\
\text { of laser } \\
\text { sessions }\end{array}$ \\
\hline $\begin{array}{llll}\text { All patients } n=56 \\
\text { Laser only } n=27\end{array}$ & 3.9 & $63 \%(n=35)$ & 4.9 & $23 \%(n=13)$ \\
$\begin{array}{l}\text { Laser plus topical antifun- } \\
\text { gals } n=29\end{array}$ & 3.6 & $56 \%(n=15)$ & 5.1 & $26 \%(n=7)$ \\
\hline
\end{tabular}


$(n=42)$ of all patients. "Microscopic cure" (complete cure) was achieved in $16 \%(n=9)$ of all patients.

For patients treated with laser only, "cultural cure" was achieved in $63 \%$ after 5.4 laser sessions as compared to $86 \%$ of nails treated with laser plus topical antifungals after 4.8 laser sessions (Table 2). "Microscopic cure" (complete cure) was achieved in 11\% of nails treated with laser only after 4.7 laser sessions, as compared to $21 \%$ of nails treated with laser plus topical antifungals after 4.0 sessions (Table 3).

\section{Discussion}

The role of non-ablative laser therapy for the treatment of onychomycosis is discussed controversially. Published data and conclusions range from effective [25] to mostly ineffective [23, 24]. This heterogeneity of published data goes in line with a big heterogeneity in treatment protocols (wavelength and total energy applied, combination of treatments, number and duration of therapy) as well as methods of evaluation and assessed endpoints (e.g. physician or patient assessment, standardized photography, mycological cure, clinical improvement, clinical cure, and Onychomycosis Severity Index). This makes a direct comparison of results and the drawing of distinct conclusions difficult $[15,23,25-34]$. In particular, the clinical efficacy of multimodal approaches, applying laser and pharmacological therapies, remains ill defined.

Patients included in our analysis were treated with amorolfine or ciclopiroxolamine, lacquers or ointments. Amorolfine is a topical antifungal that inhibits Delta14sterol reductase and cholestenol Delta-isomerase, thereby depleting ergosterol and causing a permeability of the fungal cell membrane. In an open-label, randomized, multicenter and controlled study conducted on 71 onychomycotic patients, treatment with amorolfine $5 \%$ lacquer after chemical nail avulsion with $40 \%$ urea showed clinical cure in $10.3 \%$ after 24 weeks and in $11.9 \%$ after 48 weeks. Complete cure comprising both negative direct microscopy and negative culture was achieved in $12.7 \%$ of all treated nails after 36 weeks [35]. In our hands, a comparable rate of complete cure of $11 \%$ was achieved in patients treated with laser only. However, $21 \%$ of patients treated with topical antifungals plus laser therapy achieved complete cure, indicating significant, additive effects.
Ciclopiroxolamine (ciclopirox) is a synthetic hydroxypyridine. Unlike most antifungals currently available, ciclopirox does not affect sterol biosynthesis. Instead, it involves chelation of polyvalent cations (such as $\mathrm{Fe}^{+}$) with inhibition of metal-dependent enzymes responsible for the degradation of toxic peroxides in the fungal cell [36]. In a trial involving a total of 71 onychomycotic patients, the application of ciclopirox $8 \%$ lacquer after chemical nail avulsion for 24 weeks led to a $17.4 \%$ and after 48 weeks to a $42 \%$ clinical cure rate in onychomycosis. After 36 weeks, negative direct microscopy and negative culture were achieved in $36.6 \%$ of all treated nails [35]. Whereas these rates of complete cure significantly exceed the results observed in our analysis, it must be noted that a relevant number of 29 patients treated with a combination of topical antifungals and laser therapy in our hands had undergone a pharmacologic antifungal therapy (topical amorolfine or ciclopiroxolamine) for 24 weeks or longer without observing any significant improvement prior to laser therapy. Hence, our collective of patients could be characterized as more challenging and less responsive to pharmacologic measures as compared to therapy-naïve patients assessed in respective pharmacologic studies.

Reported rates for complete cure rates achieved by oral antifungals range between 55 and $78 \%$, as reported by a cumulative meta-analysis by Gupta and colleagues [37]. These include terbinafine $250 \mathrm{mg}$ daily for 3-4 months with $76-78 \%$ cure, itraconazole $400 \mathrm{mg}$ in a three- to four-pulse regimen with $63-75 \%$ cure, and continuous intake of itraconazole $200 \mathrm{mg}$ with a cure rate of $59-63 \%$. The intake of griseofulvin $500-1000 \mathrm{mg}$ daily up to 18 months is associated with a cure rate of $55-60 \%$, whereas fluconazole $150 \mathrm{mg}$ weekly for 3-12 months achieves a cure in only 48-53\% [37]. Yet, even though these rates exceed results achieved for topical antifungals, lasers or combinations thereof, it is obvious that systemic therapy does not guarantee healing and, as noted before, in the daily practice systemic therapy is limited by contraindications, adverse events or simply refusal by patients [38-41].

With regard to the efficacy of laser therapy, in 2014, Raulin et al. performed a single-blind study comparing laser treatment with a 1064-nm long-pulsed and a short-pulsed Nd:YAG laser on onychomycotic toenails.

\footnotetext{
(See figure on next page.)
}

Fig. 1 Clinical courses of hallux toenails treated with laser only or laser plus topical antifungals. Displayed are representative images of four patients before (left panel: $\mathbf{a}, \mathbf{d}, \mathbf{f}, \mathbf{h}$ ) and after (right panel: $\mathbf{c}, \mathbf{f}, \mathbf{g}, \mathbf{i})$ treatment with a 1.064-nm diode laser only $(\mathbf{a}-\mathbf{c}$ ) or laser plus topical antifungals $(\mathbf{d}$-i). Individual representative courses show $\mathbf{b}$ clinical improvement after 36 weeks and 6 sessions and $\mathbf{c}$ complete cure after 68 weeks and 11 sessions with laser only (negative culture and microscopy); e clinical improvement after 30 weeks and 5 sessions of laser plus topical antifungals (negative culture and microscopy); $\mathbf{g}$ clinical improvement after 20 weeks and 5 sessions of laser plus topical antifungals (negative culture, positive microscopy); i clinical improvement after 24 weeks and 6 sessions of laser plus topical antifungals (negative culture, positive microscopy) 


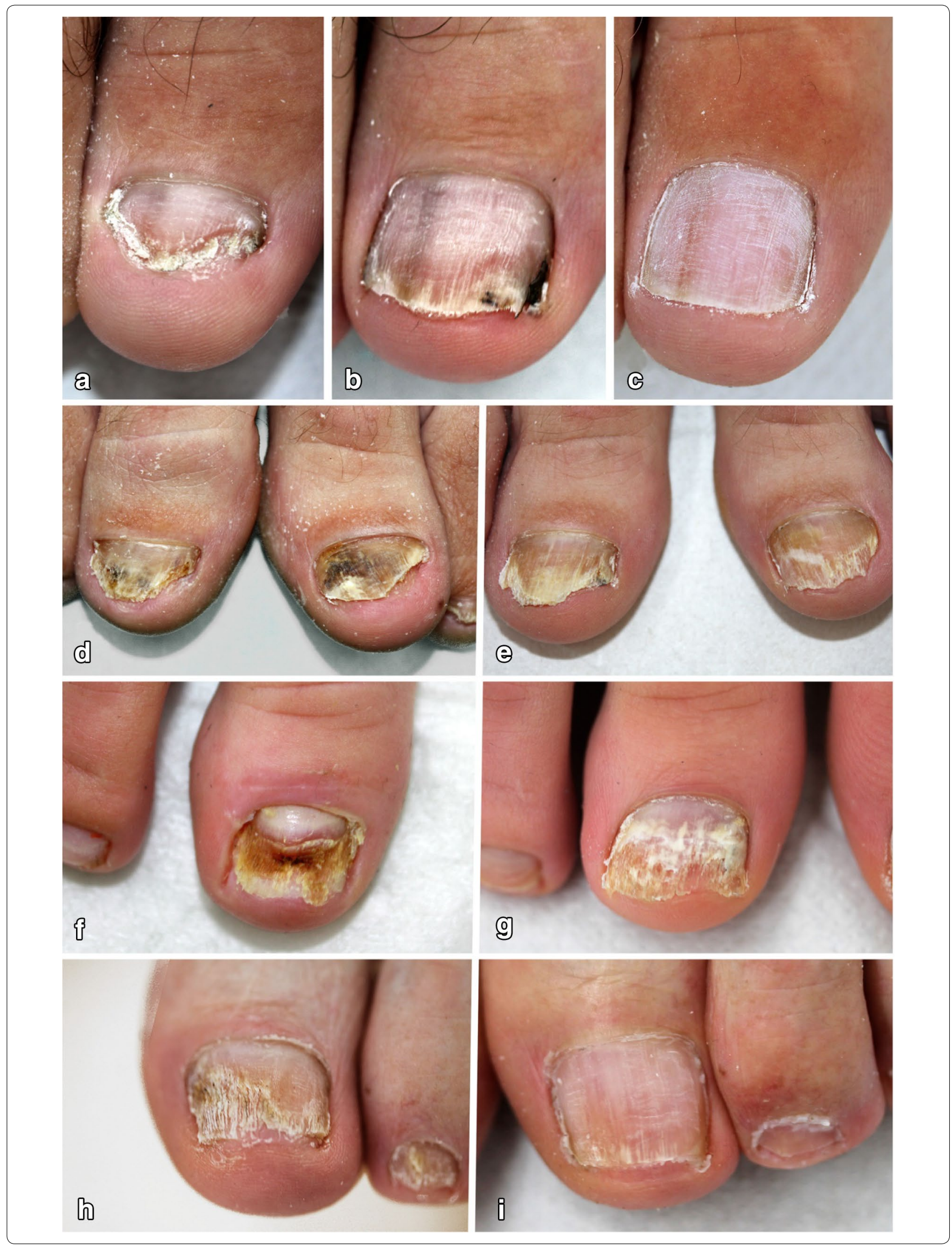


Table 2 Cultural clearance after laser plus/minus topical antifungals for the treatment of onychomycosis

\begin{tabular}{llll}
\hline & $\begin{array}{l}\text { Pretherapeutic } \\
\text { positive }\end{array}$ & $\begin{array}{l}\text { Posttherapeutic } \\
\text { positive }\end{array}$ & $\begin{array}{l}\text { Average } \\
\text { of laser } \\
\text { sessions }\end{array}$ \\
\hline $\begin{array}{l}n=56 \\
\begin{array}{l}\text { Laser only } \\
n=27\end{array}\end{array}$ & $100 \%(n=56)$ & $25 \%(n=14)$ & 4.9 \\
$\begin{array}{l}\text { Laser plus topi- } \\
\text { cal antifun- } \\
\text { gals }\end{array}$ & $100 \%(n=29)$ & $37 \%(n=10)$ & 5.4 \\
$n=29$ & $14 \%(n=4)$ & 4.8 \\
\hline
\end{tabular}

Table 3 Microscopic clearance after laser plus/minus topical antifungals for the treatment of onychomycosis

\begin{tabular}{llll}
\hline & $\begin{array}{l}\text { Pretherapeutic } \\
\text { positive }\end{array}$ & $\begin{array}{l}\text { Posttherapeutic } \\
\text { positive }\end{array}$ & $\begin{array}{l}\text { Average } \\
\text { of laser } \\
\text { sessions }\end{array}$ \\
\hline$n=56$ & $100 \%(n=56)$ & $84 \%(n=47)$ & 4.2 \\
$\begin{array}{l}\text { Laser only } \\
n=27\end{array}$ & $100 \%(n=27)$ & $89 \%(n=24)$ & 4.7 \\
$\begin{array}{l}\text { Laser plus topi- } \\
\text { cal antifun- } \\
\text { gals } \\
n=29\end{array}$ & & $79 \%(n=23)$ & 4 \\
\hline
\end{tabular}

At 9-month follow-up after two laser sessions the rate of complete cure in both groups was $20 \%$. Negative fungal culture could be reached in $70 \%$ with the short-pulsed laser, compared with $60 \%$ with the long-pulsed laser [42]. Surprisingly, in a study performed by the same group in 2017, after 12 months no mycological, microscopic or even cosmetic improvement was reported for 82 onychomycotic nails treated four times with a short-pulsed 1064-nm Nd:YAG laser at intervals of 4-6 weeks [24]. The 1064-nm diode laser systems assessed in our analysis were also used in a clinical study performed by Renner and colleagues. Herein, 82 onychomycotic nails were treated with the respective 1064-nm diode laser as well as in combination with topical antifungals. Clinical efficacy was assessed using the Onychomycosis Severity Index (OSI) and showed an improvement of $25 \%$. The treatment protocol did not include microscopic or cultural analyses of fungal pathogens [25].

Our results presented here show that treatment of onychomycosis using laser only does indeed have an effect, with a cultural cure rate of up to $63 \%$ (Table 2). This efficacy could be, furthermore, improved in combination with topical antifungals achieving up to $86 \%$ of cultural cure. Also patients that did not benefit from a prior topical pharmacologic antifungal therapy showed an improvement in combination with laser therapy.
Surprisingly, 26\% (7 of 27) patients showed a clinical cure after six laser treatments with laser only, whereas laser treatment in combination with topical antifungals resulted in a lower percentage of cure of $21 \%$ (6 of 29 patients); yet, this result was achieved after only 4.3 laser treatments (Table 1). This observation suggests that the efficacy of laser therapy is positively correlated to the overall number of laser treatments, and hence the outcome of therapy can be improved by increasing the number of laser sessions. Accordingly, we advise our patients to undergo at least three laser sessions before the individual response is evaluated. If a clinical improvement has been achieved up to this point, a continuation of laser therapy can be suggested until complete cure or until no further improvement may be observed.

Our results also show that out of a total of 56 patients 29 patients had been applying topical antifungals for 24 weeks or longer prior to the start of laser therapy without any significant effect. In combination with laser therapy eventually $69 \%$ of these 29 patients achieved clinical improvement and $21 \%$ achieved a complete clinical cure. While this effect could also be attributed to the sole continuation of pharmacologic measures, the timely correlation to the start of laser therapy suggests that mycological cure in these cases is indeed rather the result of combination therapy. Adverse effects of laser therapy for onychomycosis reported in the literature range from pain up to tissue necrosis [24, 43]. However, if performed responsibly it can be considered as safe and effective, as highlighted by our experience presented here. Finally, we can state from our experience that positive clinical results strongly correlated with the patients' overall satisfaction with the treatment.

\section{Conclusions}

Our results show that the application of a 1.064-nm diode laser is effective for the treatment of onychomycosis caused by dermatophytes achieving complete cure in $11 \%$ of the patients. Treatment efficacy can be, furthermore, increased by combining laser therapy with topical antifungals reaching up to $21 \%$ of complete cure. This multimodal approach is of particular interest for patients that are limited by contraindications against systemic antifungals. If performed responsibly laser therapy causes minimal to no side effects and comes along with a high patient satisfaction. In our hands, the overall efficacy of the therapy positively correlated with the total number of laser sessions.

Prospective, ideally intra-individually half-side controlled trials with relevant numbers of patients are still urgently needed to establish better treatment protocols and to support the proposed relevance of a multimodal, 


\section{pharmaco-physical approach for the management of onychomycosis.}

\section{Authors' contributions}

PAG, GW, PF and BAB carried out the studies, participated in collecting data, and drafted the manuscript. EB performed the statistical analysis and participated in its design. EB, HS BAB, PAG and $\mathrm{HB}$ helped to draft the manuscript. All authors read and approved the final manuscript.

\section{Author details}

${ }^{1}$ Department of Dermatology, Heinrich-Heine-University, 40225 Düsseldorf, Germany. ${ }^{2}$ Department of Radiation Oncology, Heinrich-Heine-University, 40225 Düsseldorf, Germany.

\section{Competing interests}

The authors declare that they have no competing interests.

\section{Availability of data and materials}

All data and materials can be found in the material section or can be accessed via Dr. Weber and Prof. Dr. A. Gerber.

\section{Consent for publication}

Every author has given consent for publication.

\section{Ethics approval and consent to participate}

This study was approved by the local ethics committee.

\section{Funding}

Not applicable.

\section{Publisher's Note}

Springer Nature remains neutral with regard to jurisdictional claims in published maps and institutional affiliations.

Received: 12 July 2018 Accepted: 7 September 2018

Published online: 24 October 2018

\section{References}

1. Ghannoum MA, Hajjeh RA, Scher R, et al. A large-scale North American study of fungal isolates from nails: the frequency of onychomycosis, fungal distribution, and antifungal susceptibility patterns. J Am Acad Dermatol. 2000;2000:641-8.

2. Sigurgeirsson B, Baran R. The prevalence of onychomycosis in the global population - a literature study. JEADV. 2014;2014:1480-91.

3. Gupta AK, Nakrieko K-A. Molecular determination of mixed infections of dermatophytes and nondermatophyte molds in individuals with onychomycosis. J Am Podiatr Med Assoc. 2014;2014:330-6.

4. Scher R, Rich P, Elewski B, Pariser D. The epidemiology, etiology, and pathophysiology of onychomycosis. Semin Cutan Med Surg. 2013;2013:S2-4.

5. Scherer WP, McCreary JP, Hayes WW. The diagnosis of onychomycosis in a geriatric population: a study of 450 cases in South Florida. J Am Podiatr Med Assoc. 2001;2001:456-64.

6. Finch JJ, Warshaw EM. Toenail onychomycosis: current and future treatment options. Dermatol Ther. 2007;20:31-46.

7. Elewski B. Onychomycosis: pathogenesis, diagnosis, and management. Clin Microbiol Rev. 1998;11:415-29.

8. Schemer A. Update: medical treatment of onychomycosis. Dermatol Ther 2012;2012:582-93.

9. AWMF. Onychomykose, ICD-10 Ziffer B35.1. Leitlinien der Deutschen Dermatologischen Gesellschaft und der Deutschsprachigen Mykologischen Gesellschaft; 2006.

10. Brenner MA, Harkless LB, Mendicino RW, Page JC. Ciclopirox $8 \%$ nail lacquer topical solution for the treatment of onychomycosis in patients with diabetes, A multicenter, open-label study. J Am Podiatr Med Assoc 2007;97:195-202.
11. Curanail 5\% nail lacquer (amorolfine hydrochloride) PL 10590/0049. http://www.mhra.gov.uk/home/groups/par/documents/websiteresource s/con2023787.pdf. MHRA PAR 2017. Accessed 01 Dec 2017.

12. Verrier J, Bontems O, Baudraz-Rosselet F, Monod M. Oral terbinafine and itraconazole treatments against dermatophytes appear not to favor the establishment of Fusarium spp. in nail. Dermatology. 2014;228:225-32.

13. Gupta AK, Uro M, Cooper EA. Onychomycosis therapy, past, present, future. J Drugs Dermatol. 2010;2010:1109-13.

14. FDA. Medical devices and clinical trial design for the treatment or improvement in the appearance of fungally-infected nails. Guidance for Industry and Food and Drug Administration Staff (FDA). March 7, 2016. http://www.fda.gov/downloads/MedicalDevices/DeviceRegulationandG uidance/GuidanceDocuments/UCM431312.dpf. Accessed 02 Dec 2017.

15. Gupta AK, Versteeg SG. A critical review of improvement rates for laser therapy used to treat toenail onychomycosis. JEADV. 2017;31:1111-8.

16. Vural E, Winfield HL, Shingleton AW, Horn TD, Shafirstein G. The effects of laser irradiation on Trichophyton rubrum growth. Lasers Med Sci. 2008;2008:349-53.

17. Wanitphakdeedecha R, Thanomkitti K, Bunyaratavej S, Manuskiatti W. Efficacy and safety of 1064-nm Nd:YAG laser in treatment of onychomycosis. J Dermatol Treat. 2016;2016:75-9.

18. Hashimoto T, Blumenthal HJ. Survival and resistance of Trichophyton mentagrophytes arthrospores. Appl Environ Microbiol. 1978;35:274-7.

19. Carney C, Cantrell W, Warner J, Elewski B. Treatment of onychomycosis using a submillisecond 1064-nm neodymium:yttrium-aluminum-garnet laser. J Am Acad Dermatol. 2013;2013:578-82.

20. Landthaler M, Haina D, Brunner R, Waidelich W, Braun-Falco O. Effects of argon, dye, and $\mathrm{Nd}$ :YAG lasers on epidermis, dermis, and venous vessels. Lasers Surg Med. 1986;1986:87-93.

21. Gupta AK, Simpson FC, Heller DF. The future of lasers in onychomycosis. J Dermatol Treat. 2016:27:167-72.

22. Anderson RR, Parrish JA. Selective photothermolysis: precise microsurgery by selective absorption of pulsed radiation. Science. 1983:220:524-7.

23. Hees H, Raulin C, Bäumler W. Laser treatment of onychomycosis: an in vitro pilot study. J Dtsch Dermatol Ges. 2012;2012:913-8.

24. Karsai S, Jäger M, Oesterhelt A, et al. Treating onychomycosis with the short-pulsed 1064-nm-Nd:YAG laser: results of a prospective randomized controlled trial. JEADV. 2017:2017:175-80.

25. Renner R, Grüsser K, Sticherling M. 1,064-nm diode laser therapy of onychomycosis: results of a prospective open treatment of 82 toenails. Dermatology. 2015;2015:128-34.

26. Hollmig ST, Rahman Z, Henderson MT, Rotatori RM, Gladstone H, Tang JY. Lack of efficacy with 1064-nm neodymium:yttrium-aluminum-garnet laser for the treatment of onychomycosis: a randomized, controlled trial. J Am Acad Dermatol. 2014;2014:911-7.

27. Landsman AS, Robbins AH. Treatment of mild, moderate, and severe onychomycosis using 870- and 930-nm light exposure. J Am Podiatr Med Assoc. 2012;102:169-71.

28. Landsman AS, Robbins AH, Angelini PF, et al. Treatment of mild, moderate, and severe onychomycosis using 870- and 930-nm light exposure. J Am Podiatr Med Assoc. 2010;100:166-77.

29. Waibel J, Wulkan AJ, Rudnick A. Prospective efficacy and safety evaluation of laser treatments with real-time temperature feedback for fungal onychomycosis. J Drugs Dermatol. 2013;2013:1237-42.

30. Rivers JK, Vestvik BJ, Berkowitz J. Real-world efficacy of 1064-nm $\mathrm{Nd}$ :YAG laser for the treatment of onychomycosis. J Cutan Med Surg. 2017;2017:108-13.

31. Okan G, Tarikci N, Gokdemir G. The effect of long-pulsed Nd:YAG laser for the treatment of onychomycosis. J Am Podiatr Med Assoc. 2017:2017:54-9.

32. Ortiz AE, Truong S, Serowka K, Kelly KM. A 1,320-nm Nd:YAG laser for improving the appearance of onychomycosis. Dermatol Surg. 2014;40:1356-60

33. Xu Y, Miao X, Zhou B, Luo D. Combined oral terbinafine and long-pulsed 1,064-nm Nd:YAG laser treatment is more effective for onychomycosis than either treatment alone. Dermatol Surg. 2014:40:1201-7.

34. Li Y, Yu S, Xu J, Zhang R, Zhao J. Comparison of the efficacy of long-pulsed $\mathrm{Nd}$ :YAG laser intervention for treatment of onychomycosis of toenails or fingernails. J Drugs Dermatol. 2014;2014:1258-63.

35. Paul C, Coustou D, Lahfa M, et al. A multicenter, randomized, open-label, controlled study comparing the efficacy, safety and cost-effectiveness of 
a sequential therapy with RV4104A ointment, ciclopiroxolamine cream and ciclopirox film-forming solution with amorolfine nail lacquer alone in dermatophytic onychomycosis. Dermatology. 2013;2013:157-64.

36. Gupta AK, Fleckman P, Baran R. Ciclopirox nail lacquer topical solution $8 \%$ in the treatment of toenail onychomycosis. J Am Acad Dermatol. 2000;43:S70-80.

37. Gupta AK, Ryder JE, Johnson AM. Cumulative meta-analysis of systemic antifungal agents for the treatment of onychomycosis. Br J Dermatol. 2004:150:537-44.

38. Elewski B, Tavakkol A. Safety and tolerability of oral antifungal agents in the treatment of fungal nail disease: a proven reality. Ther Clin Risk Manag. 2005;2005:299-306.

39. Okamoto J, Fukunami M, Kioka H. Frequent premature ventricular contractions induced by itraconazole. Circ J. 2007;2007:1323-5.
40. Bangsgaard N, Saunte DM, Folkenberg M, Zachariae C. Serious adverse events reporting on systemic terbinafine: a Danish register-based study. Acta Derm Venereol. 2011;2011:358-9.

41. Ahmad SR, Singer SJ, Leissa BG. Congestive heart failure associated with itraconazole. Lancet. 2001;2001:1766-7.

42. Hees H, Jäger MW, Raulin C. Treatment of onychomycosis using the 1064 nm Nd:YAG laser: a clinical pilot study. J Dtsch Dermatol Ges. 2014;2014:322-9.

43. Leverone AP, Guimarães DA, Bernardes-Engemann AR, Orofino-Costa R. Partial necrosis of the hallux in a patient treated with laser for onychomycosis, is this procedure really worthwhile? Dermatol Surg. 2015;41:869-72.
Ready to submit your research? Choose BMC and benefit from:

- fast, convenient online submission

- thorough peer review by experienced researchers in your field

- rapid publication on acceptance

- support for research data, including large and complex data types

- gold Open Access which fosters wider collaboration and increased citations

- maximum visibility for your research: over $100 \mathrm{M}$ website views per year

At BMC, research is always in progress.

Learn more biomedcentral.com/submissions 